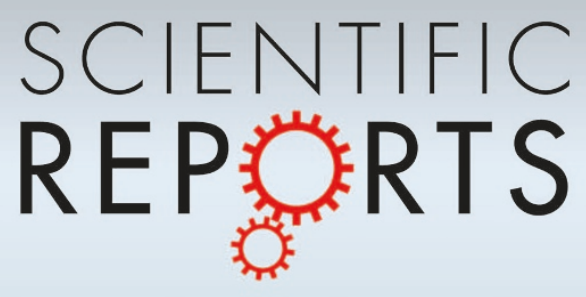

OPEN

SUBJECT AREAS:

SPECTROSCOPY

NANOPHOTONICS

APPLIED PHYSICS

ATOMIC AND MOLECULAR PHYSICS

Received

29 May 2012

Accepted

16 August 2012

Published

11 September 2012

Correspondence and requests for materials should be addressed to H.X.X. (hxxu@iphy.ac.

cn)

\section{In-situ plasmon-driven chemical reactions revealed by high vacuum tip-enhanced Raman spectroscopy}

\author{
Mengtao Sun' ${ }^{1}$ Zhenglong Zhang ${ }^{1,2}$, Hairong Zheng' ${ }^{2}$ \& Hongxing Xu'
}

\begin{abstract}
'Beijing National Laboratory for Condensed Matter Physics, Institute of Physics, Chinese Academy of Sciences, P. O. Box 603-146, Beijing, 100190, People's Republic of China, ${ }^{2}$ College of Physics and Information Technology, Shaanxi Normal University, Xi'an, 710062, People's Republic of China.
\end{abstract}

With strong surface plasmons excited at the metallic tip, tip-enhanced Raman spectroscopy (TERS) has both high spectroscopic sensitivity and high spatial resolution, and is becoming an essential tool for chemical analysis. It is a great challenge to combine TERS with a high vacuum system due to the poor optical collection efficiency. We used our innovatively designed home-built high vacuum TERS (HV-TERS) to investigate the plasmon-driven in-situ chemical reaction of 4-nitrobenzenethiol dimerizing to dimercaptoazobenzene. The chemical reactions can be controlled by the plasmon intensity, which in turn can be controlled by the incident laser intensity, tunneling current and bias voltage. The temperature of such a chemical reaction can also be obtained by the clearly observed Stokes and Anti-Stokes HV-TERS peaks. Our findings offer a new way to design a highly efficient HV-TERS system and its applications to chemical catalysis and synthesis of molecules, and significantly extend the studies of chemical reactions.

U sing a sharp metal tip as a controlled plasmon antenna to excite the localized surface plasmons and consequently enhance the electromagnetic field in the vicinity of the tip apex, tip-enhanced Raman spectroscopy (TERS) has become an essential analytical tool to give an extremely high optical sensitivity $^{1-8}$. Moreover, since the enhanced Raman signals are from the very tiny tip area with the size of tens of nanometers, TERS can offer a nanoscale resolution beyond the diffraction limit of light as well. Since high vacuum can give a clean chemical environment useful for chemical analysis, it is essential to build up TERS in a high vacuum system to achieve a novel solution for chemical analysis. However, it is very challenging to combine the optics with high vacuum systems to achieve highly efficient illumination and collection of extremely weak Raman signals.

Recently, it was found that surface plasmons, which are collective oscillation of free electrons at a dielectricmetal interface $e^{9,10}$, play an important role in chemical reactions ${ }^{11-24}$, such as photochromic reactions ${ }^{11}$, polymerization ${ }^{12}$, isomerization ${ }^{13}$, dissociation ${ }^{14}$ and catalytic reactions ${ }^{15-24}$. Since the sharp metal tip in TERS can create a "hot site" to excite strong surface plasmon resonances at the tip, and both high spectroscopic sensitivity and nanoscale resolution can supply definitive in-situ spectroscopic evidences for chemical reactions, TERS can be an ideal tool to investigate and control plasmon-driven chemical reactions.

\section{Results}

The design of HV-TERS is shown in Figure 1. The Raman optical system is connected to high vacuum by a stainless steel bellow valve, where a stainless steel tube was sealed inside the bellow with one end equipped with a quartz window to allow the light in and out of the high vacuum chamber, and the other end was equipped with a long working distance objective $(\mathrm{NA}=0.5)$ to allow a highly efficient excitation and collection of weak Raman signals at the STM tip. The objective is in the high vacuum chamber, and the focus can be located in the nanogap in the tip-substrate junction. The outside of the bellow is fixed to the Raman probe equipped with two Notch filters to block the scattered light at the laser frequency. The Raman probe is mounted on a $3 \mathrm{D}$ adjuster equipped with piezo motor stages for optical fine-alignment.

By exploiting the advantages of our HV-TERS setup, we first measured HV-TERS of 4-nitrobenzenethiol (4NBT) adsorbed on a silver film (see Figure $2 \mathrm{a}$ and $2 \mathrm{~b}$ ) on the different bias voltage of $-0.5 \mathrm{~V}$ and $+0.5 \mathrm{~V}$, respectively. For comparison, we also measured the normal Raman spectrum of 4NBT powder (see Figure 2c). It was found that the strongly enhanced Raman peaks are in the region of 1000 to $1700 \mathrm{~cm}^{-1}$, and the TERS 


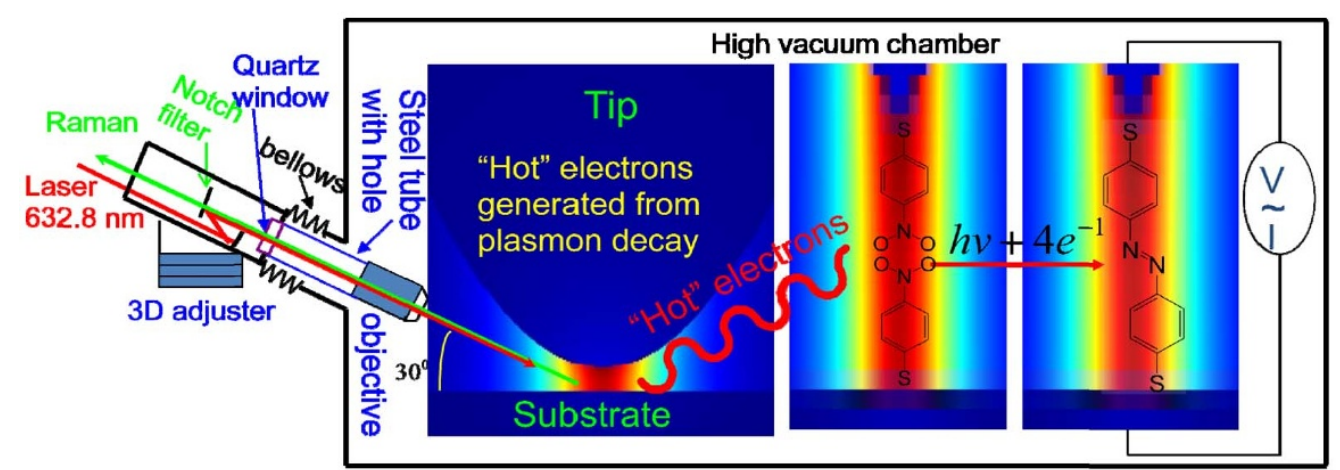

Figure $1 \mid$ The schematics of HV-TERS. Plasmon-driven chemical reaction measured in HV-TERS.

spectrum is significantly different from the normal Raman spectrum of 4 NBT in this region. Comparing Figure $2 \mathrm{a}$ and Figure $2 \mathrm{~b}$, we can see that both profiles are almost the same, which reveals that the polarity of voltage does not influence the TERS profile. Comparing Figure $2 a-b$ with Figure $2 c$, we can see that the profiles are significantly different. The Raman intensity of $-\mathrm{NO}_{2}$ stretching mode of 4 NBT at $1336 \mathrm{~cm}^{-1}$ in Figure 2c has almost disappeared in Figure 2a and $2 \mathrm{~b}$. Furthermore, the strongly enhanced Raman peaks at 1142, 1387 and $1432 \mathrm{~cm}^{-1}$ in Figure $2 \mathrm{a}$ and $2 \mathrm{~b}$ cannot be observed in Figure 2c. We also found that these TERS are the same as the SERS spectra of dimercaptoazobenzene (DMAB) converted from p-aminothiophenol (PATP) ${ }^{15,16}$, and the Raman peaks in Figure $2 \mathrm{a}$ and $2 \mathrm{~b}$ were assigned. The strongly enhanced vibrational mode of $\mathrm{ag}_{17}$ at $1432 \mathrm{~cm}^{-1}$ in Figure $2 \mathrm{a}$ and $2 \mathrm{~b}$ is the $-\mathrm{N}=\mathrm{N}$ - stretching vibrational mode of DMAB. So, DMAB cannot only be produced from PATP ${ }^{15,16}$, but also from 4 NBT by a plasmon-driven surface catalyzed reaction. To further confirm that the measured TERS is DMAB converted from 4NBT, we compared HV-TERS (Figs. 2d and 2e) with normal Raman spectrum of 4 NBT powder (Figure $2 \mathrm{f}$ ) in the region of 700 to $1000 \mathrm{~cm}^{-1}$. It was found that the strong Raman peaks of $4 \mathrm{NBT}$ at $854 \mathrm{~cm}^{-1}$ in Figure $2 \mathrm{f}$ cannot be observed in the TERS in Figs. $2 \mathrm{~d}$ and 2e, whereas several strongly enhanced TERS peaks in Figs. 2d and 2e cannot be observed in Figure 2f. Theoretical calculations revealed that there are four kinds of vibrational modes in this region. Raman-active ag modes (see Figure S1 in supplementary information) can be clearly observed in Figure $2 \mathrm{~d}$ and $2 \mathrm{e}$. Note the bg modes can also been observed in Figure $2 \mathrm{~d}$ and $2 \mathrm{e}$, though the
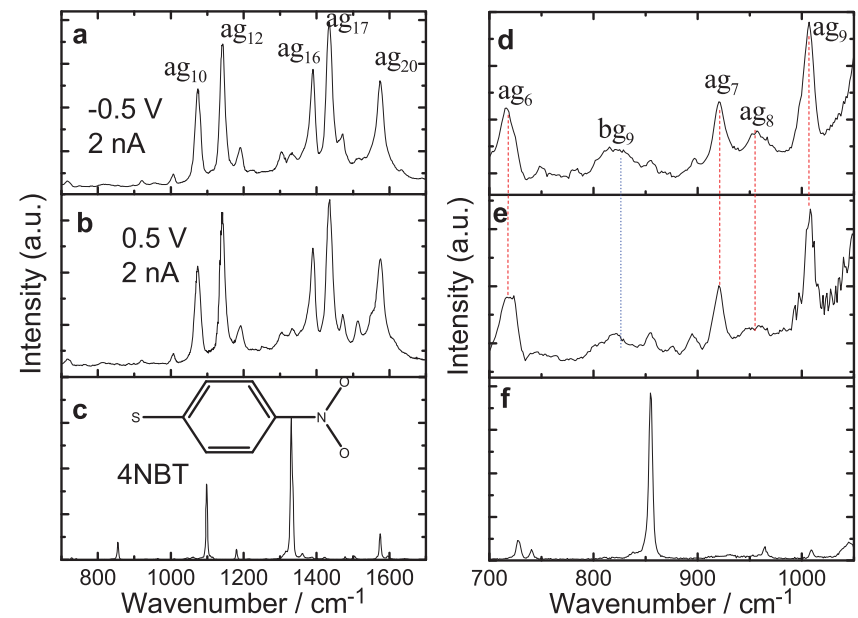

Figure $2 \mid$ HV-TERS measurement of plasmon-driven chemical reaction. (a) and (b) the measured HV-TERS of DMAB in the region of 700 to $1700 \mathrm{~cm}^{-1}$, and (c) the normal Raman spectrum of 4NBT powder. (d)-(f) are the zoom-in spectra of (a)-(c) from 700 to $1050 \mathrm{~cm}^{-1}$. simulation in Figure S1 is weak. It is worthwhile to note that the TERS peaks of DMAB on Ag films are so strongly enhanced that the very weak Raman peaks in the low Raman frequency region in the normal SERS can be clearly observed in more detail. The good agreement between the experiment and the simulated (ag modes) Raman spectra in the low frequency region provides additional spectroscopic evidence for the conversion of 4NBT to DMAB.

With the advantage of our HV-TERS, we can perform in-situ measurements of plasmon-driven chemical reactions to observe how these occur. We measured Raman spectra of 4 NBT in the HV-TERS system with different incident laser power. Figure $3 \mathrm{a}$ is the TERS of $4 \mathrm{NBT}$ at low laser power $(0.5 \%$ or $\sim 10 \mu \mathrm{W}$ at the sample); three main peak positions of the measured TERS above $1000 \mathrm{~cm}^{-1}$ are the same as the normal Raman spectrum of 4NBT powder in Figure 2c. With the increase of the incident laser intensity, we found that there was almost no change of the TERS peaks below $3 \%$ of laser intensity (see Figure $3 \mathrm{~b}$ ). When the laser intensity is larger than $3 \%$, the measured TERS revealed that 4 NBT molecules can be gradually dimerized to DMAB. Figure $3 \mathrm{c}$ is the TERS at $10 \%$ of laser intensity. It is found that the $4 \mathrm{NBT}$ and DMAB coexist, since the fingerprint vibrational peak of $4 \mathrm{NBT}$ at $1336 \mathrm{~cm}^{-1}$ for the $-\mathrm{NO}_{2}$

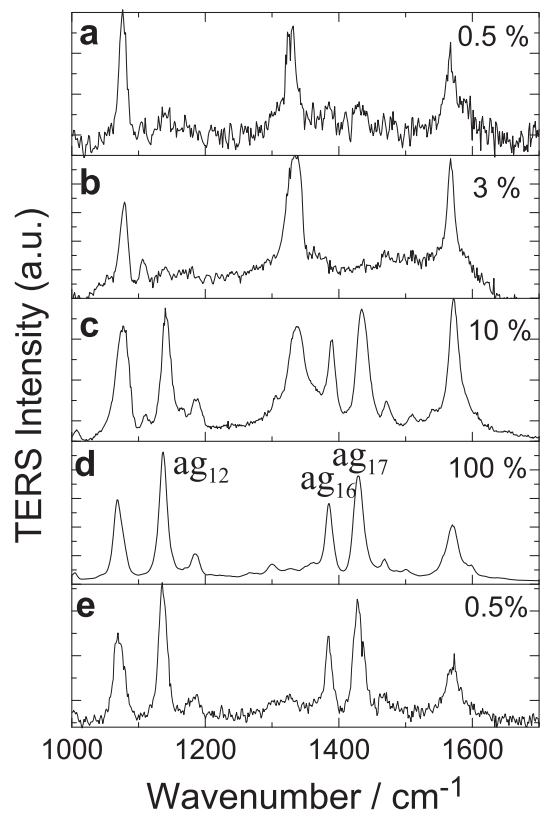

Figure 3 Laser intensity controlled dynamics of plasmon-driven chemical reactions. Laser power at (a) $0.5 \%$, (b) $3 \%$, (c) $10 \%$, (d) $100 \%$ and (e) $0.5 \%$ at bias voltage $1 \mathrm{~V}$, and current $1 \mathrm{nA}$. The vibrational modes of $\mathrm{ag}_{12}, \mathrm{ag}_{16}$ and $\mathrm{ag}_{17}$ for DMAB were assigned in (d). 


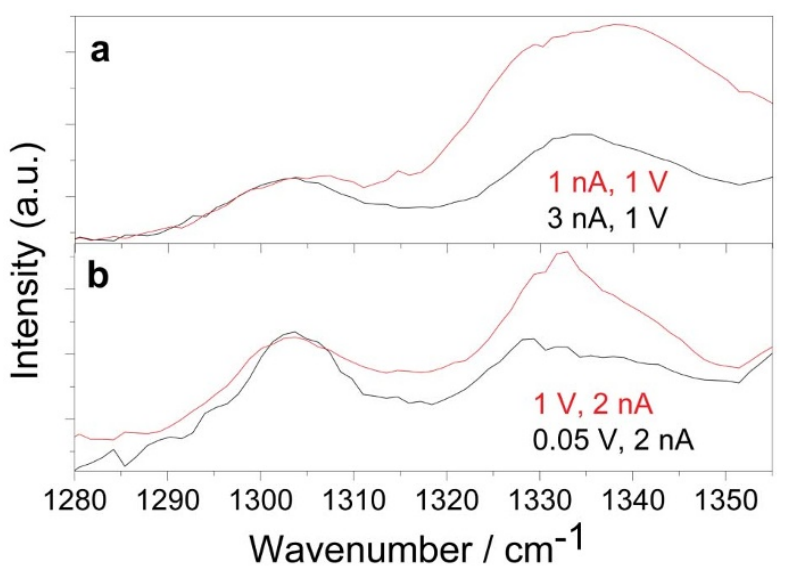

Figure $4 \mid$ The current and voltage dependent plasmon-driven chemical reaction. (a) the current and (b) the voltage dependence of TERS of DMAB. The Raman intensity were normalized at $1304 \mathrm{~cm}^{-1}$ for comparison.

stretching mode of 4NBT (Figure S2 in supplementary information) still exists, but decreases relative to the peak at $1575 \mathrm{~cm}^{-1}$ of benzene ring stretching vibration with the increase of the laser intensity. When the laser intensity is increased to $100 \%$, almost all 4 NBT molecules were dimerized to $\mathrm{DMAB}$, since the vibrational peak of $4 \mathrm{NBT}$ at $1336 \mathrm{~cm}^{-1}$ almost totally disappeared (see Figure 3d). Figure 3e shows the measured TERS with low laser power, again the same as that in Figure 3a; it is found that the TERS profile in Figure $3 \mathrm{e}$ is identical as that in Figure 3d, except for the difference of the Raman intensity, while not back to Figure 3a. Figure 3d and 3e clearly revealed that this chemical reaction of dimerization really happened.

In our HV-TERS system, we can control the tunneling current and bias voltage to change the distance between the metallic tip and the substrate, thus change the local electromagnetic field enhancement. The influences of the control of the tunneling current and bias voltage should somehow be equal to the change of the incident laser intensity. Figure 4a revealed that with an increase of the tunneling current at a constant bias voltage, resulting in a decrease of the distance between the tip and the substrate, the Raman peak at $1336 \mathrm{~cm}^{-1}$ decreases. The decrease of the gap distance can increase the local electromagnetic field, and excite stronger surface plasmon resonances at the metallic tip region ${ }^{8}$. Similar to the increase of the incident laser intensity, more dimerization conversions of $4 \mathrm{NBT}$ to DMAB assisted by stronger plasmon resonances can be obtained. Meanwhile, less 4NBT remaining results in the decrease of the fingerprint Raman peak at $1336 \mathrm{~cm}^{-1}$, as observed. It should be noted that the increase of the tunneling current could also promote the chemical reaction, since the chemical bonds could be selectively broken or formed by the tunneling electron current only ${ }^{25}$. But as indicated in Figure 3a, no obvious chemical reaction is observed at a low laser power for a long time illumination at a rather significant tunneling current in the order of $\mathrm{nA}$, which indicates the contribution from the tunneling current would be ignored for our case here. Similarly, we measured voltage dependent TERS when the current is fixed to $2 \mathrm{nA}$. From Figure $4 \mathrm{~b}$, as we can see, with the decrease of the bias voltage, the Raman peak at $1336 \mathrm{~cm}^{-1}$ decreases for the same reason as for the current dependence. Since the distance between the tip and the substrate decreases due to the decreased bias voltage, the plasmon resonances become stronger. The stronger the surface plasmon resonances, the more "hot" electrons are generated by plasmon decay, and better chemical reactions can be obtained experimentally.

We also experimentally studied the vibrational bending mode of $-\mathrm{NO}_{2}$ at $854 \mathrm{~cm}^{-1}$ in TERS of DMAB versus the tunneling current

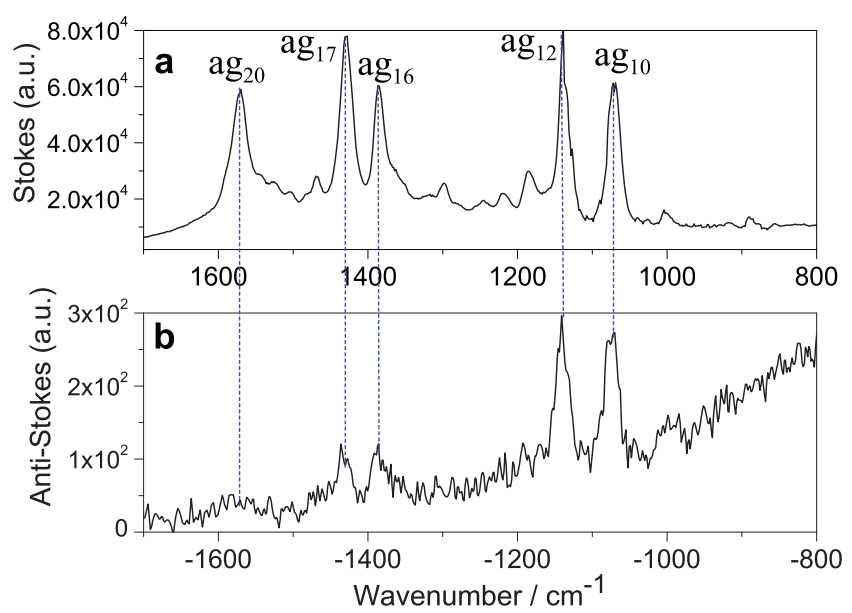

Figure $5 \mid$ The Stokes and anti-Stokes HV-TERS peaks. (a) The Stokes TERS, and (b) the Anti-Stokes TERS at the bias voltage $1 \mathrm{~V}$ and the tunneling current $2.5 \mathrm{nA}$.

and bias voltage (see Figure $\mathrm{S} 3$ in supplementary information). These are qualitatively consistent with those in Figure $4 \mathrm{a}$ and $4 \mathrm{~b}$, respectively. It is worthwhile to note that the plasmon-driven chemical reactions in HV-TERS are much faster than those in SERS ${ }^{17}$. In SERS studies, the vibrational mode of $4 \mathrm{NBT}$ at $1336 \mathrm{~cm}^{-1}$ was not noticeably decreased until after 140 minutes, whereas in HV-TERS, it can decrease greatly within several minutes. The narrow nanogap region in the sharp tip/metal substrate geometry can supply extremely large gap plasmon resonances that benefit the plasmon-driven chemical reactions.

Finally, the anti-Stokes HV-TERS measurement again confirms that 4NBTs were dimerized to DMAB (see Figure 5), in which the $\mathrm{ag}_{10}, \mathrm{ag}_{12}, \mathrm{ag}_{16}$ and $\mathrm{ag}_{17}$ vibrational modes of DMAB were clearly observed experimentally. Furthermore, the $-\mathrm{NO}_{2}$ vibrational modes of $4 \mathrm{NBT}$ at $-854 \mathrm{~cm}^{-1}$ and $-1336 \mathrm{~cm}^{-1}$ disappeared, which means that the probability of this plasmon-driven chemical reaction is very high. It is very important to know accurately the experimental temperature of the plasmon-driven chemical reaction, which can be fitted with Eq. 1,

$$
I_{s} / I_{a s}=a \times e^{\left(\hbar \omega / k_{B} T\right)}
$$

where $I_{s}$ and $I_{a s}$ are the intensities of Stokes and anti-Stokes, and $\hbar, k_{B}$ and $T$ are the Planck constant and Boltzmann constant, experimental temperature, respectively, and $a$ is an experimental constant. The fitted experimental temperature is $327( \pm 4) K$, and the experimental parameter $a$ in Eq. (1) is $2.06( \pm 0.19)$.

\section{Discussion}

The HV-TERS system used here supplies an ideal configuration with a sharp metal tip coupled to the metal substrate to excite surface plasmons. Although photochemical reactions could still happen at the absorption resonance, it is unlikely for the 4NBT molecules used here. Both the adsorption of the 4NBT molecules and the adsorption of the compound of $4 \mathrm{NBT}$ on silver film are in the UV region, far away from the excitation in the red used here. Surface plasmons can decay to "hot" electrons with a kinetic energy above the Fermi level ${ }^{26,27}$, which have a key role for the chemical reaction in the TERS system as revealed in Figure 3 where a stronger laser intensity generates a stronger plasmon resonance. Strong plasmon resonances produce a higher density of "hot" electrons generated from plasmon decay. The "hot" electrons with high kinetic energy above the Fermi level could jump to the unoccupied resonant energy level of chemical reactants near the metal surface. The occupation of a resonance level by a "hot" electron changes the equilibrium potential energy surface 
from the neutral situation to a temporal negative ion situation of the composite adsorbate molecule. The energy above the Fermi level of the "hot" electrons can be transferred to the intra molecular vibrational energy during the interactions. In both cases the energy barrier of the chemical reactions could be decreased ${ }^{19}$. Since four electrons are needed to produce one DMAB from two 4NBTs, a higher density of "hot" electrons induced by stronger incident laser intensity and stronger local plasmon resonances can boost the chemical reaction. The chemical reaction is unlikely to happen when the plasmon intensity is too weak when the incident laser power is too low, as observed in Figure 3. Thermal effects during the plasmon-driven chemical reaction can be largely ruled out according to no obvious temperature increase by fitting the Stokes and anti-Stokes HV-TERS as shown in Figure 5.

\section{Methods}

Vibrational spectra were measured with a home-built HV-TERS setup as shown in Figure 1. The diameter and height of the cylindrical vacuum chamber are 25 and $28 \mathrm{~cm}$, respectively. The pressure in the vacuum chamber is about $10^{-7} \mathrm{~Pa}$. A gold tip with the radius of about $50 \mathrm{~nm}$ was made by electrochemical etching of a $0.25 \mathrm{~mm}$ diameter gold wire ${ }^{28}$. The substrate was prepared by evaporating $100 \mathrm{~nm}$ silver film to a newly cleaned mica film under high vacuum. The film was immersed in a $1 \times 10^{-5} \mathrm{M}$ 4NBT ethanol solution for 24 hours, and then washed with ethanol for 10 minutes to guarantee that there was only one monolayer of 4NBT molecules adsorbed on the silver film. Then the sample was immediately put into the high vacuum chamber. To get a good signal-to-noise ratio, the TERS signals were collected with an acquisition time of 40 seconds and accumulated 20 times for each spectrum. We also measured normal Raman scattering spectrum of 4 NBT powder, using Leica microscopy equipment in a confocal Raman spectroscopic system (Renishaw, Invia), and the incident wavelength is $632.8 \mathrm{~nm}$.

1. Stöckle, R. M., Suh, Y. D., Deckert, V. \& Zenobi, R. Nanoscale chemical analysis by tip-enhanced Raman spectroscopy. Chem. Phys. Lett. 318, 131-136 (2000).

2. Hayazawa, N., Inouye, Y., Sekkat, Z. \& Kawata, S. Detection and characterization of longitudinal field for tip-enhanced Raman spectroscopy. Opt. Commun. 183, 333-336 (2000).

3. Anderson, M. S. Locally enhanced Raman spectroscopy with an atomic force microscope. Appl. Phys. Lett. 4, 3130-3132 (2000).

4. Domke, K. F., Zhang, D. \& Pettinger, B. Toward Raman fingerprints of single dye Molecules at atomically smooth $\mathrm{Au}(111)$. J. Am. Chem. Soc. 128, 14721-14727 (2006).

5. Bailo, E. \& Deckert, V. Tip-enhanced Raman scattering. Chem. Soc. Rev. 37, 921-930 (2008).

6. Steidtner, J. \& Pettinger, B. High-resolution microscope for tip-enhanced optical processes in ultrahigh Vacuum. Rev. Sci. Instr. 78, 103104 (2007).

7. Jiang, N. et al. Observation of multiple vibrational modes in ultrahigh vacuum tipenhanced Raman spectroscopy combined with molecular-resolution scanning tunneling microscopy. Nano Lett. (DOI:10.1021/nl2039925).

8. Sun, M. T., Fang, Y. R., Yang, Z. L. \& Xu, H. X. Chemical and electromagnetic mechanisms of tip-enhanced Raman scattering. Phys. Chem. Chem. Phys. 11, 9412-9419 (2009)

9. Ritchie, R. H. Plasma losses by fast electrons in thin films. Phys. Rev. 106, 874-881 (1957).

10. Brockman, J. M., Nelson, B. P. \& Corn, R. M. Surface plasmon resonance imaging measurements of ultrathin organic films. Annu. Rev. Phys. Chem. 51, 41-63 (2000).

11. Tsuboi, Y., Shimizu, R., Shoji, T. \& Kitamura, N. Near-infrared continuous-wave light driving a two-photon photochromic reaction with the assistance of localized surface Plasmon. J. Am. Chem. Soc. 131, 12623-13267 (2009).

12. Deeb, C. et al. Plasmon-based free-radical photopolymerization: effect of diffusion on nanolithography processes. J. Am. Chem. Soc. 133, 10535-10542 (2011).

13. Hubert, C. et al. Near-field photochemical imaging of noble metal nanostructures. Nano Lett. 5, 615-619 (2005).
14. Chen, C. J. \& Osgood, R. M. Near-field photochemical imaging of noble metal nanostructures. Phys. Rev. Lett. 50, 1705 (1983).

15. Fang, Y., Li, Y., Xu, H. X. \& Sun, M. T. Ascertaining p, p'-Dimercaptoazobenzene Produced from p-Aminothiophenol by Selective Catalytic Coupling Reaction on Silver Nanoparticles. Langmuir 26, 7737-7746 (2010).

16. Huang, Y. F. et al. When the signal is not from the original molecule to be detected: chemical transformation of para-aminothiophenol on Ag during the SERS measurement. J. Am. Chem. Soc. 132, 9244-9246 (2010).

17. Dong, B., Fang, Y. R., Chen, X. W., Xu, H. X. \& Sun, M. T. Substrate-, wavelength-, and time-dependent plasmon-assisted surface catalysis reaction of 4-nitrobenzenethiol dimerizing to $\mathrm{p}, \mathrm{p}^{\prime}$-dimercaptoazobenzene on $\mathrm{Au}, \mathrm{Ag}$, and $\mathrm{Cu}$ films. Langmuir 27, 10677-10682 (2011).

18. Zhu, H., Ke, X, Yang, X., Sarina, S. \& Liu, H. Reduction of nitroaromatic compounds on supported gold nanoparticles by visible and ultraviolet light. Angew. Chem. Int. Ed. 49, 9657 (2010).

19. Christopher, P., Xin, H. \& Linic, S. Visible-light-enhanced catalytic oxidation reactions on plasmonic silver nanostructures. Nature Chem. 3, 467-472 (2011)

20. Xie, W., Herrmann, C., Kömpe, K., Haase, M. \& Schlücker, S. Synthesis of bifunctional Au/Pt/Au Core/Shell nanoraspberries for in situ SERS monitoring of Platinum-catalyzed reactions. J. Am. Chem. Soc. 133, 19302-19305 (2011).

21. Navalon, S., de Miguel, M., Martin, R., Alvaro, M. \& Garcia, H. Enhancement of the catalytic activity of supported gold nanoparticles for the Fenton reaction by light. J. Am. Chem. Soc. 133, 2218-2226 (2011).

22. Silva, C. G., Juárez, R., Marino, T., Molinari, R. \& García, H. Influence of excitation wavelength (UV or visible light) on the photocatalytic activity of titania containing gold nanoparticles for the generation of hydrogen or oxygen. J. Am. Chem. Soc. 133, 595-602 (2011).

23. Wadayama, T. \& Yokawa, M. Hot-electron assisted reaction of $p$-nitrobenzoic acid adsorbed on metal-insulator-metal tunnel junction's electrode surface. Chem. Phys. Lett. 428, 348-351 (2006).

24. Sun, M. T. \& Xu, H. X. A novel application of plasmonics: plasmon driven surface catalyzed eeactions. Small 2012, in press (DOI: 10.1002/smll.201200572).

25. Lee, H. J. \& Ho, W. Single-bond formation and characterization with a scanning tunneling microscope. Science 286, 1719-1722 (1999).

26. Knight, M. W., Sobhani, H., Nordlander, P. \& Halas, N. J. Photodetection with active optical antennas. Science 332, 702-704 (2011).

27. Moskovits, M. Hot electrons cross boundaries. Science 332, 676-677 (2011).

28. Ren, B., Picardi, G. \& Pettinger, B. Preparation of gold tips suitable for tipenhanced Raman spectroscopy and light emission by electrochemical etching. Rev. Sci. Instrum. 75, 837-841 (2004).

\section{Acknowledgments}

This work was supported by the National Basic Research Project of China (Grants 2009CB930701, 2009СB930704 and 2007CB936804) and the National Natural Science Foundation of China (Grants 90923003, 10874233, 10874234 and 20703064).

\section{Author contributions}

H.X.X. and H.R.Z. supervised the project, M.T.S. and H.X.X. designed the experiments. Z.L.Z. and M.T.S. measured HV-TERS experimentally. M.T.S. and H.X.X. analyzed data and wrote the paper.

\section{Additional information}

Supplementary information accompanies this paper at http://www.nature.com/ scientificreports

Competing financial interests: The authors declare no competing financial interests.

License: This work is licensed under a Creative Commons

Attribution-NonCommercial-ShareAlike 3.0 Unported License. To view a copy of this license, visit http://creativecommons.org/licenses/by-nc-sa/3.0/

How to cite this article: Sun, M., Zhang, Z., Zheng, H. \& Xu, H. In-situ plasmon-driven chemical reactions revealed by high vacuum tip-enhanced Raman spectroscopy. Sci. Rep. 2 , 647; DOI:10.1038/srep00647 (2012). 